\title{
The 3D Surface Measurement and Simulation for Turbine Blade Surface Based on Color Encoding Structural Light
}

\author{
Yang Ling and Yuan Jiahe \\ The Higher Educational Key Laboratory for Measuring \& Control Technology and \\ Instrumentations of Heilongjiang Province, Harbin University of Science and \\ Technology, Harbin, China, \\ lindaleg9@126.com
}

\begin{abstract}
This paper proposes a color encoding structural light approach for the three-dimensional measurement of turbine blade surface. It adopts RGB color mode design coding scheme, by using the six basic colors, red, green, blue, yellow, magenta, and cyan for color-coded stripes. Every three stripes are grouped together, while the color combinations for each group are different. The coding pattern based on these color stripes is projected to the blade. A CCD camera then captures the image modulated by the blade. We then decode this image to obtain the three-dimensional coordinates of the measured points on blade surface. According to the structure light measurement principle, we designed and determined the parameters of the three-dimensional measurement. This paper established a mathematical model of a turbine blade through simulation by using $3 d$ s max software. We designed a threedimensional measurement system for the simulation, and obtained the three-dimensional coordinates of the blade surface by measuring the turbine blade. Finally, the threedimensional blade model reconstructed by the measured data is established.
\end{abstract}

Keywords: 3D measurement; color encoding; structural light; turbine blade

\section{Introductions}

Currently, China's hydropower industry is booming, and it has widely served in many areas of the national economy. During the turbine operation, due to the influence of various complex underwater environments, turbine blades are eroded, appeared pitting, and even partial peeling can happen. These conditions can easily lead to resonate and other adverse reactions, loss of the turbine's efficiency, and resulting in the insufficient power output. Thus, the measurement and maintenance of key components of hydraulic equipment has become the foci of many researches. Since turbine blades are composed of very complex free-form surfaces, it is very difficult to be measured. Also note that due to the complex external environment as the consequence of long-time submersion underwater, the demands for maintenance personnel labor is intensive. Therefore, many hydropower stations use welding robot to repair eroded blades [1]. The research presented in this paper on the 3D surface measurement can provides a theoretical basis for robotic welding.

There exist various different methodologies for measuring the turbine blade. This paper proposes a color-coded structural light measurement technique based on a machine vision technology, for measuring the turbine blade. There are two steps: in the first step, various types of two-dimensional light information are obtained through an observation system. In the second step, the three-dimensional structural information contained via two-dimensional lighting is processed by an algorithm and a model can be reconstructed. 


\section{Structural Light Measurement Principle}

Structural light measurement method [2-3] is a frequently used method of measuring. Structural light measurement generally uses the measurement systems composed of a device which is capable of producing structural light, an object to be measured, and an image receiving equipment. This method is based on the well-known principle of triangulation, i.e., given two angles and one side; the other sides can be solved mathematically. This is also called "active triangulation". There exist various methods for 3D measurement with structural light, e.g., point structural light method, linear structural light method, and coded structural light method, etc. 3D measurement is widely used. For example, the measurement and repair of turbine blade depend on it.

Point structural light method is the simplest and most basic method [4]. However, it suffers from a number of disadvantages. First of all, point-by-point scanning must be performed in order to acquire the contour information of the entire blade surface. Secondly, the three-dimensional coordinate of each point is determined by formula, and hence carries a heavy calculation burden. Last but not the least, it results in sharp increased demands for time in image acquisition, processing capacity and image processing time, which in turn seriously affects the measuring efficiency. These drawbacks can be addressed by the linear structural light measurement method. In this method, since points become lines, the information for all points on a line can be obtained by scanning the blade surface. Compared with the point structural light method, the measuring efficiency can be greatly improved. However, the line light source must constantly change its projection angle so that the surface of the entire blade within the field of view can be scanned. MA large number of images must be taken to obtain three-dimensional coordinates of all points. Therefore, the measurement efficiency still is still less than desirable.

Following the idea of transforming points into lines, a line can be further projected into a two-dimensional pattern on the blade. By encoding the patters in certain ways, it becomes the coded pattern method [5-7]. Coded structural light measurement is one of the most reliable three-dimensional measurement techniques.

\section{Color Stripe Encoding and Decoding Principle}

Compared with geometric features, the color features are easier to identify. Therefore, using color images for image recognition can have good results. Since the three basic colors in the RGB system, namely red, green, and blue, can be easily identified during the image processing, RGB color is used. This color stripe coding method uses the change of the channels of red (R), green $(\mathrm{G})$, blue $(\mathrm{B})$ and the superposition on each other to get a variety of colors. RGB color mode uses the RGB model to assign a value of intensity 0-255 to RGB components for each pixel in the image. For example, for red color, $\mathrm{R}$ component has a value of 255, while both $\mathrm{G}$ and $\mathrm{B}$ components have value of 0 . In our experiment, we only assign two values, 0 or 255 for each color in order to maximize the difference between colors, which in turn yields the best recognition performance. This also improves interference-resistance ability. Coding patterns is generated with 6 colors: red, green, blue, and their complements, cyan, magenta and yellow.

Based on the above analysis, the values of R, G, B components for each color is obtained, as shown in Table 1.

Table 1. The Values of Different Colors in RGB Mode

\begin{tabular}{l|l|l|l|l|l|l}
\hline color & red & green & blue & yellow & magenta & cyan \\
\hline
\end{tabular}




\begin{tabular}{l|l|l|l|l|l|l}
\hline$R$ & 255 & 0 & 0 & 255 & 255 & 0 \\
\hline$G$ & 0 & 255 & 0 & 255 & 0 & 255 \\
\hline$B$ & 0 & 0 & 255 & 0 & 255 & 255 \\
\hline
\end{tabular}

In this process, each color is represented as a stripe. We then combine 6 different color stripes in such a way that

1) Every three adjacent color stripes form a group,

2) The order of all colors among all the stripes cannot be repeated, for example, Such as "cyan, green, blue"; "green, blue, magenta"; "blue, magenta, red"; "magenta, red, yellow"; "red, yellow, red"; "yellow, red, red"; "red, red, green"; "red, green, cyan"; "green, cyan, yellow".

3) The width of each stripe is preferably 2 pixels.

Since interferences among the color stripes may occur due to the light intensity, we deliberately placed a black stripe between all color stripes. The width of the black stripe is set to be 4 pixels, as shown in Figure 1.

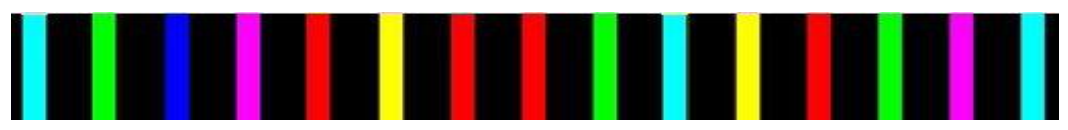

Figure 1. The Coding for Combinations of the Color Stripes

There are in total $6^{3}-(6-1)=211$ groups of eligible combinations. Thus, the number of color stripes being used is 213 .

There are various combinations of the color stripes. Different initial color choices lead to different coding combinations of the color stripes. After many tests, we found that the only way to get longest period of the code combinations is to set red, red, red as the initial color stripe, and all other colors being in ordered. Figure 2 shows the resulted coding pattern for combinations of the color stripes.

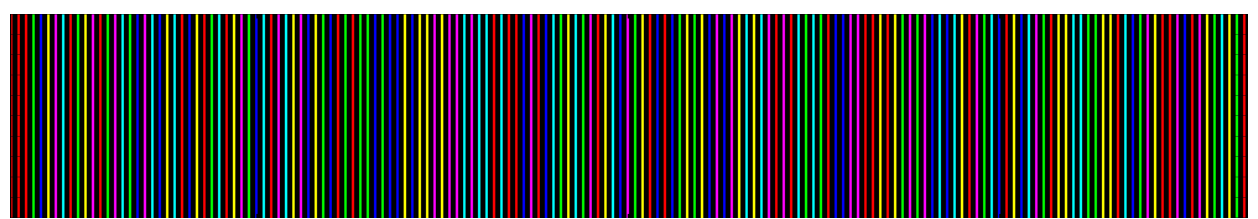

Figure 2. The Coding Pattern for Combinations of the Color Stripes

\section{Building the Turbine Blade Model}

We first model the turbine blade in 3ds max software. As shown in Figure 3, we establish a rectangular area in the front view. The number of segments of the length, width, and height are properly set. This is to make the rectangle subdivided so that each part can be edited. We then enter the edit panel, select "FFD $4 \times 4 \times 4$ " editor on the Edit command panel, and use rotation, translation, and other functions to edit the control point, so that the model of the blade is in a preliminary stage. Finally, we choose "Mesh Smooth" editor on the Edit command panel to make the surface of the blade model smooth. We next open the Material Editor to edit the material of the blade surface. Since turbine blades are generally made of stainless steel, we choose the metal pattern in the material editor so that the blade model has a metallic sheen. In the "specular highlights" adjustment panel, we set the high light level to 
238 , and the gloss to 23 , close the self-luminous option and set the opacity to $100 \%$. Figure 4 is a rendered graph of the blade model being attached with materials.

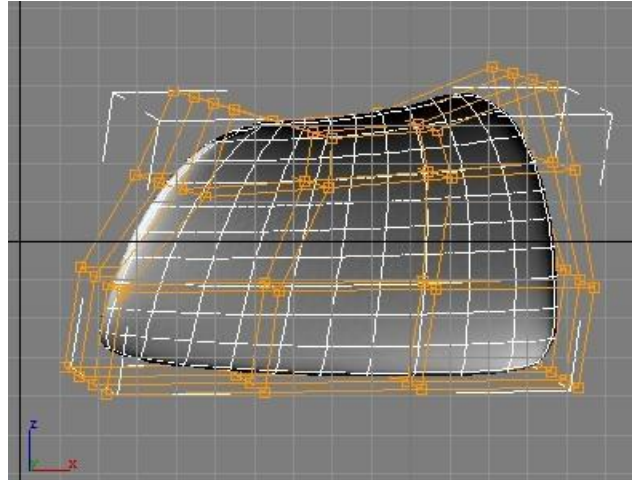

Figure 3. Modeling the Turbine Blade

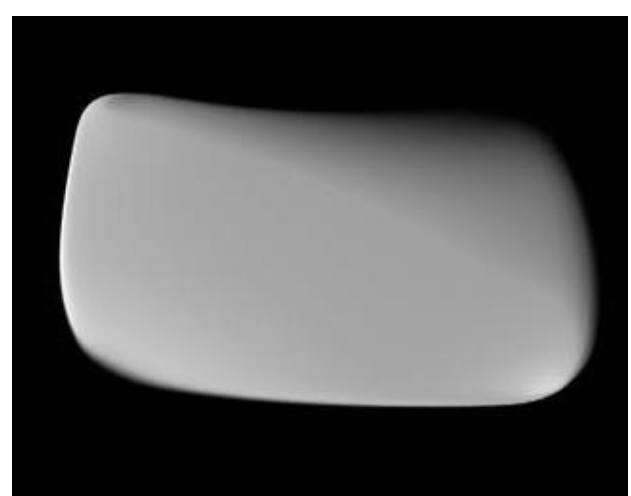

Figure 4. Rendered Graph of Turbine Blade

\subsection{Setting up the Projector}

First, the projectorshouldbe set up according to the principle of structural light projection, as shown in Figure 5.

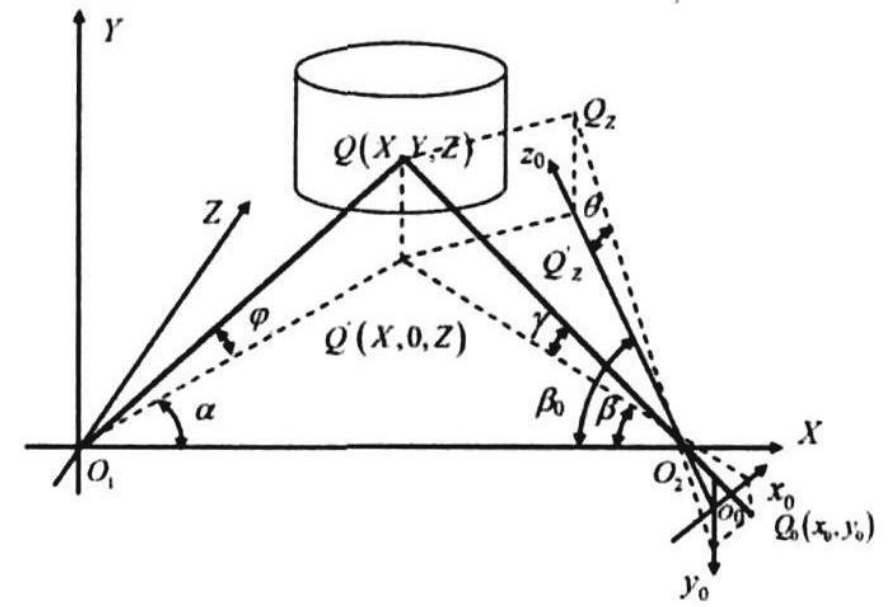

Figure 5. Point Principle Structural Light Method

The triangle $\Delta O_{1} O_{2} Q$ in the figure is consists of the center points of the projection lamp $\left(Q_{1}\right)$, the blade model (Q )and the camera lens $\left(O_{2}\right)$. Let $O_{1} O_{2}=B$. The point $\mathrm{Q}$ is located in the object coordinate system $X Y Z O_{1}$, and the image point $Q_{0}$ formed on image sensor is located in the image coordinate system $x_{0} o_{0} y_{0}$ with coordinates $\left(x_{0}, y_{0}\right)$, thus $x_{0} o_{0} z_{0}$ and $X O_{1} Z$ are in the same plane. The object point $Q$ is located on $X O_{1} Z$ plane、 $y_{0} o_{0} z_{0}$ plane. Its projections on $o_{0} z_{0}$ axis are $Q^{\prime}, Q_{Z}$ and $Q_{Z}^{\prime}$, respectively. $\beta_{0}$ is the angle betweenthe camera Optical axis and the $\mathrm{X}$ axis. The projection angle $\alpha$ can be computed according to the color decoding. 
Using Figure 5 and the principle of triangulation, we can establish the following:

$$
\begin{gathered}
Z=\frac{B}{\cot \alpha+\cot \beta} \\
X=Z \cot \alpha \\
\tan \theta=\frac{y_{0}}{f}=\frac{Y}{O_{2} Q_{Z}^{\prime}} \\
\cos \left(\beta_{0}-\beta\right)=\frac{O_{2} Q_{Z}^{\prime}}{O_{2} Q^{\prime}} \\
\tan \left(\beta_{0}-\beta\right)=\frac{x_{0}}{f} \\
\sin \beta=\frac{Z}{O_{2} Q^{\prime}}
\end{gathered}
$$

From the above equations, we can further derive the following formulas

$$
\begin{gathered}
Z=\frac{B}{\cot \alpha+\frac{x_{0}+f \cot \beta_{0}}{f-x_{0} \cot \beta_{0}}} \\
Y=\frac{Z \cdot y_{0}}{f \sin \beta_{0}-x_{0} \cos \beta_{0}} \\
X=Z \cot \alpha
\end{gathered}
$$

In the above equations, $f 、 B 、 \beta_{0}$ are all calculated from given parameters. For example, $f$, the focal length of the camera lens, is given. $\beta_{0}$, the angle between the optical axis of the camera and the $X$ axis, is a predetermined angle $B$, the distance between the center of the spotlight and the center of the camera lens, is also known. The coordinates $\left(\mathrm{x}_{0}, \mathrm{y}_{0}\right)$ of the image point $Q_{0}$ of the point $\mathrm{Q}$ is known, the projection angle $\alpha$ can be obtained from the color decoding. Thus, by plugging these known parameters into equations (1)-(3), we can obtain the three-dimensional coordinate values $\mathrm{X}, \mathrm{Y}, \mathrm{Z}$ of the point $\mathrm{Q}$. The depth of the blade in the projection field of view can be obtained by changing the projection angle along the directions of $\varphi$ and $\alpha$.

The above three formulas for computing the values $\mathrm{X}, \mathrm{Y}, \mathrm{Z}$ are the basic formulas of structural light method, and is also the basis for three-dimension measurements of the structural light.

We then set up the projector accordingly, with the simulation projector replaced by a target spotlight, as shown in Figure 6. Let us place the spotlight in the origin $O_{1}$ in the world coordinate system. i.e., the center of the projection system has coordinates $(0,0,0)$. Also note that the angle between the optical axis and the x-axis is $60^{\circ}$, i.e., $\alpha_{0}=60^{\circ}$, and the projection angle is $60^{\circ}$, i.e., $\alpha_{1}=30^{\circ}$. We next set up the spotlight according to the parameters determined earlier. Light intensity coefficient is $20 \mathrm{~cd}$. The color of the light projected by the spotlight is white, since only white light can minimally affect the projected color-coded patterns; the starting and the ending point of the near attenuation of target spotlight are set to $100 \mathrm{~mm}$ and $900 \mathrm{~mm}$, respectively; the starting and the ending point of the far attenuation of target spotlight are set to $2000 \mathrm{~mm}$ and $5000 \mathrm{~mm}$, respectively. 


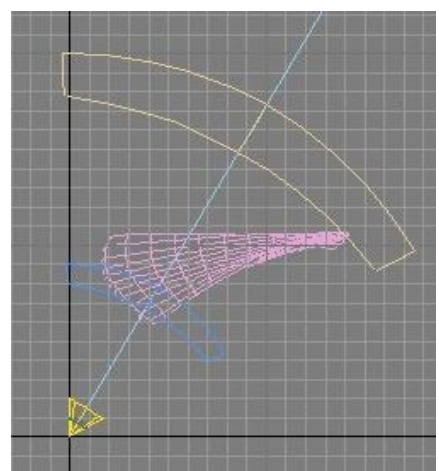

Figure 6. The Set-up of the Projector

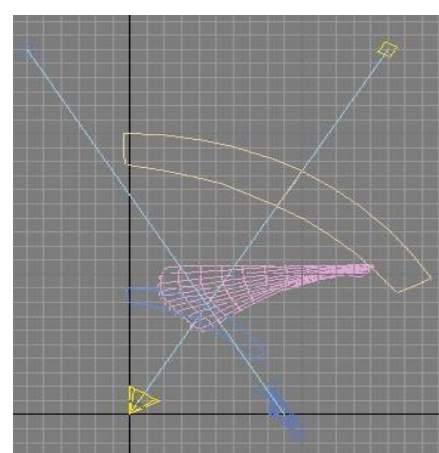

Figure 7. The Set-up of the Video Camera

The simulation video camera is placed at a point on $\mathrm{x}$ axis whose distance to the projector is $900 \mathrm{~mm}$. Thus the center point of the lens, $O_{2}$, has the coordinate of $(900,0,0)$, as shown in Figure 7.The angle between the optical axis and the $\mathrm{x}$-axis is $60^{\circ}$, i.e., $\beta_{0}=60^{\circ}$. The opening angle of the projector is $60^{\circ}$, i.e., $\alpha_{1}=30^{\circ}$.In our simulation experiment, since the blade model is rather large, the results are best if the center of the blade model is placed at the coordinate of $(800,900,24.57)$.

Due to the large size of the model, it requires two stages to measure the entire blade model. We need to measure it in two fields of view separately, then splice the images together in order to reconstruct the entire blade.

There should be two fields of views by our original design. However, for convenience, we would just translate the blade model. During our experiments, if we translated it to the left by $560 \mathrm{~mm}$ simultaneously, i.e., $\mathrm{x}$-axis coordinate of the blade model becomes 240 , the result is better. The coded pattern is projected to the turbine blade model by the simulation projector. The images are obtained by simulation video camera. The simulation system is now fully constructed.

\subsection{Simulation Results}

The coding pattern is projected to the blade. Figure 8 shows the images obtained in two fields of image.

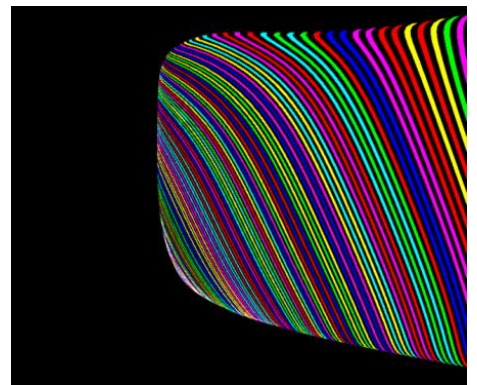

a) Coding pattern shoot in field of view 1

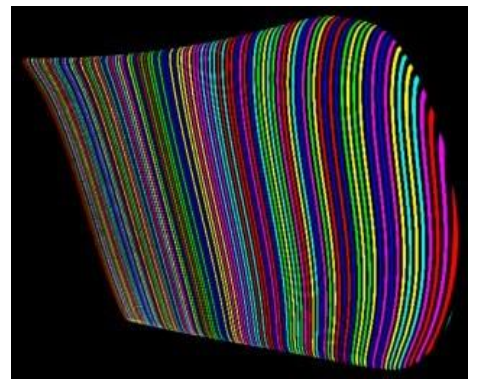

b) Coding pattern shot in field of view 2

Figure 8. Two Images of the Coding Pattern Projection 
We then decode and splice the images from the two fields of views. The results are shown in Figure 9.
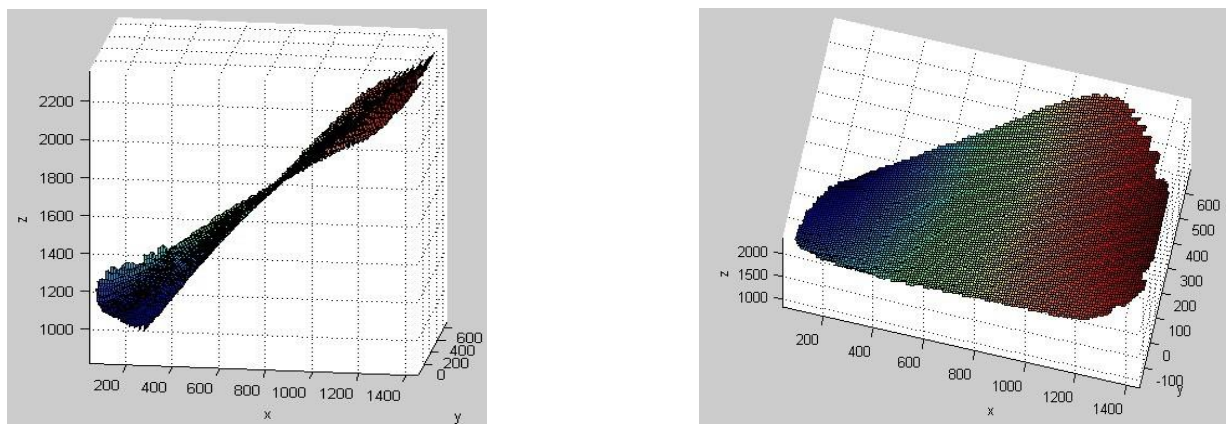

Figure 9. Reconstruction of the Turbine Blade

\section{Conclusions}

Experimental results show that the proposed three-dimensional color-coded structural light measurement method for measuring the turbine blade is feasible, easy to operate. Data processing is faster compared with the point structural light and line structural measurement methods.

\section{References}

[1] Q. Shu and D. Tang, "Path Trajectory Planning of Manipulator for Repairing Hydraulic Turbine [J]", Journal of Harbin Univerty of Science and Technology, vol. 2, (2014), pp. 16-24.

[2] X. Yu, L.-n. Shan, S.-n. Cao and H. Wu, "The Review of Structured Light Time Encoding Technologies [J]", Journal of Harbin Univerty of Science and Technology, vol. 15, (2010), pp. 98-105.

[3] A. Shang, H. Yuan and G. Qin, "The 3D measurement of large curved surface based on encoding structured light [J]", Information Technology, vol. 7, (2008), pp. 74-77.

[4] X. Yu, J. Zhang and L. Wu, "Advances in structured light 3D vision technique [J]", Journal of Astronautic Metrology and Measurement, vol. 5, (1997), pp. 43-48.

[5] J. Tajima and M. Iwakawa, "3-D Data Acquisition by Rainbow Range Finder [J]", International Conference on Pattern Recognition, (1990), pp. 309-313.

[6] S. Y. Chen and Y. F. Li, "Self-recalibration of a Colour-encoded Light System for Automated Threedimensional Measurements [J]", Measurement Science \& Technology, vol. 14, (2003), pp. 33-40.

[7] L. C. Chen, S. H. Tsai and K. C. Fan, "A New Three-dimensional Profilometer for Surface Profile Measurement Using Digital Fringe Projection and Phase Shifting [J]", Key Engineering Materials, vol. 295, (2005), pp. 471476. 
International Journal of Signal Processing, Image Processing and Pattern Recognition Vol.8, No.3 (2015) 